\title{
Transport via a quantum shuttle
}

\author{
A.D. Armour* and A. MacKinnon \\ The Blackett Laboratory, Imperial College of Science, \\ Technology and Medicine, London SW7 2BW, United Kingdom
}

(Dated: October 23, 2018)

\begin{abstract}
We investigate the effect of a quantised vibrational mode on electron tunneling through a chain of three quantum dots. The outer dots are coupled to voltage leads, but the position of the central dot is not rigidly fixed. Motion of the central dot modulates the size of the tunneling barriers in opposite ways so that electron tunneling is correlated with the position of the oscillator. We treat the electronic part of the problem using a simple Coulomb-blockade picture, and model the vibration of the central dot as a quantum oscillator. We calculate the eigenspectrum of the system as a function of the energy level shift between the outer dots. Using a density matrix method, we include couplings to external leads and calculate the steady-state current through the device. The current shows marked resonances which correspond to avoided-level crossings in the eigenvalue spectrum. When the tunneling length of the electrons is of order the zero-point position uncertainty of the quantum oscillator, current far from the electronic resonance is dominated by electrons hopping on and off the central dot sequentially; the oscillator can be regarded as shuttling electrons across the system [L.Y. Gorelik, et al., Phys. Rev. Lett. 80, 4526 (1998)]. Damping of the oscillator can increase the current by preventing electrons from hopping 'backwards'.
\end{abstract}

\section{INTRODUCTION}

Electron tunneling rates through mesoscopic junctions are strongly modulated by changes in the physical extent of the barriers which can arise from excitation of vibrational degrees of freedom. through insulating barriers has an exponential dependence on the physical extent of the barriers and so is sensitive to even relatively small changes in their size. In standard semiconductor structures the components are rigidly fixed so that fluctuations in the widths of tunnel barriers are relatively unimportant. However, whenever electron transport occurs across flexible tunnel barriers modulation of the barrier widths due to vibrations is expected to affect the tunneling. The perturbation of tunneling processes across a single mesoscopic jupftion by a mechanical degree of freedom has been analysed in some detail, and is now relatively well understood. 1 . 3 However, when there are two or more flexible junctions connected in series more complicated effects arise, in particular excitation of vibrational degrees of freedom can lead to shuttling of electrons between the junctions.

The idea of a mesoscopic electron shuttle in which a vibrational degree of freedom modulates tunneling rates across two junctions in turn was proposed recently by Gorelik et al.3 (although a fully classical charge shuttle is far from new $)$. Gorelik et al. considered a system in which electrons are transported between two leads by a small metallic grain embedded in an elastic medium. The interaction between charges on the grain and the leads couples to the position of the oscillator leading to a dynamic instability. Electrons tunnel onto the grain from one of the leads, resulting in a Coulombic repulsion which drives the grain towards the other lead where the electrons tunnel off: hence vibrations of the grain shuttle electrons between leads. In the model of Gorelik et al. the electron tunneling is treated within the orthodox theory of single electron tunneling, whilst the elastic medium is treated as a classical oscillator.

A mesosfepic shuttle device similar in some respects to that proposed by Gorelik et al. has been fabricated by Blick et al 10 The device closely resembles a miniaturised bell clapper, consisting of two tunneling contacts with a cantilever in between. However, the cantilever does not 'ring' (i.e. shuttle electrons) spontaneously, but instead is driven by strong gate voltages applied across the cantilever itself (away from the tunneling contacts). The rate of electron tunneling can be controlled by the amplitude of the drive when it is tuned to one of the natural frequencies of the cantilever. Electron transport in this system can again be understood within orthodox single electron tunneling theory, modified to account for position dependent behavior in the tunneling rates.60

Systems in which electron transport petween two contacts is mediated by a vibrational mode of a self-assembled structure have also been investigated. 8 The most striking example of such a system is the $C_{60}$ single electron transistor, fabricated by Park et al. $\mathrm{O}$ In this device, a single $C_{60}$ molecule was deposited in a narrow gap between gold electrodes. The current flowing through the device was found to increase sharply whenever the applied voltage was sufficient to excite vibrations of the molecule about the minima of the van der Waals potential between it and the electrodes, or an internal mode of the molecule itself. 6.120 .11

\footnotetext{
* Current address: School of Physics and Astronomy, University of Nottingham, Nottingham NG7 2RD, United Kingdom
} 
The device fabricated by Park et al. is an example of a molecular electronic device 22 in which electrical conduction occurs through single molecules connected to conventional leads. The junctions between molecular components and leads will be much more flexible than those in conventional solid-state nanostructures and fluctuations in their width may modify their current characteristics significantly. Furthermore, vibrational modes of the molecular components themselves may play an important role in determining the transport properties.13

Variations in the widths of tunnel barriers are also expected to have an important effect on the transport properties of nanoparticle chains 14 Nanoparticle chains consist of small metal grains stabilised by ligands, with electronic transport occurring via tunneling between the metal particles. Because of the relative softness of the ligand matrix, vibrations of the metal grains can significantly modify the electronic tunneling rates.

In the present work, electron tunneling through an electromechanical system in the extreme quantum mechanical and Coulomb blockade limits is investigated. Our interest is focused on the case where the width of the tunnel barriers for electrons are modulated by a quantum mechanical vibrational degree of freedom. We analyse a model shuttle system consisting of a row of three quantum dots in which the central dot is mounted on a quantum harmonic oscillator.

The model considered here is simplified in many respects so that it is possible to build up an understanding of the characteristics of the transport which occurs when there is strong coupling between the electronic degrees of freedom and the displacement of a quantum harmonic oscillator. We assume that the capacitance of the central grain or dot, and the outer dots is sufficiently high that transport is confined to the Coulomb blockade regime. Furthermore, we assume that the electronic states of the system are coherent. We use the language of semiconductor nanostructures, describing our system in terms of quantum dots, because it provides a convenient short-hand for what is in effect a localised electronic state. In practice, what we refer to quantum dots could be actual semiconductor dots, large molecules or metallic nanoparticles. Similarly, the oscillator could be an ultra-high frequency mechanical resonator with a dot mounted on its tip, like that studied by Blick et al., 3 alternatively the oscillator mode could arise from vibration of the central 'dot' within a stabilising elastic medium or the potential confining it between the outer dots.

We find that the current characteristics of the model shuttle can largely be understood by analysing the eigenspectrum of the isolated system of three dots and the quantum oscillator. Tunnel coupling of the dot states, to each other and to the position of the oscillator, leads to repulsion of the eigenvalues and mixing of the eigenstates associated with states localised on individual dots. The mixed states consist of superpositions of the states associated with the individual dots and hence lead to delocalisation of the electronic states between the dots. Analysis of the current which flows when the shuttle is weakly coupled to leads, reveals strong resonances corresponding to the occurrence of the delocalised states. The current through the shuttle is found to depend sensitively on the amount by which the oscillator is damped, the strength of the couplings between the dots and the background temperature. A preliminary account of some of these findings has been given elsewhere.15

The organisation of the paper is as follows. In section II we describe the details of our model shuttle system. We introduce the Hamiltonian of the system and describe how coupling to external leads can be taken into account. In section III we examine the eigenspectrum of the system as a function of the difference in energy between the levels in the fixed dots. The current characteristics of the device are described in section IV. A summary and discussion of the results is given in section V. Details of how the Hamiltonian can be approximated by a finite matrix are given in appendix $\mathrm{A}$ and the derivation of the density matrix equation of motion is outlined in appendix B.

\section{MODEL FORMULATION}

We begin by detailing the model Hamiltonian which we use to describe the behavior of the shuttle system. The shuttle consists of a linear chain of three quantum dots and a single vibrational mode, as shown schematically in Fig. 1. The physical locations of the two outer dots are fixed, while the central dot is mounted on the vibrational mode whose behavior is modelled by a quantum oscillator. The electronic part of the system is idealised: each of the dots is represented by a single, localised, energy state. The dynamics of electron transport through the system is analysed using the density matrix formalism as this allows couplings to external leads and the effects of the oscillator's environment to be incorporated most conveniently.

\section{A. Tight-binding model}

Within the Coulomb blockade regime, the charging energy of adding more than one electron to the shuttle is assumed to be sufficiently high that only one transport electron can occupy the chain of three dots at any one time. Therefore, the electronic degrees of freedom of the isolated system can be represented completely by the state in which none of the dots are occupied $16.17|0\rangle$ and the three localised states associated with the lefthand, central and 
righthand dots: $|l\rangle,|c\rangle$ and $|r\rangle$ respectively. The system is modelled by a tight-binding Hamiltonian of the form

$$
\begin{aligned}
H= & \varepsilon_{l}|l\rangle\left\langle l\left|+\varepsilon_{r}\right| r\right\rangle\left\langle r\left|+\varepsilon_{c}(\hat{x})\right| c\right\rangle\langle c|+\hbar \omega \hat{d}^{\dagger} \hat{d} \\
& -V \mathrm{e}^{-\alpha\left[x_{0}+\hat{x}\right]}(|c\rangle\langle l|+| l\rangle\langle c|) \\
& -V \mathrm{e}^{-\alpha\left[x_{0}-\hat{x}\right]}(|c\rangle\langle r|+| r\rangle\langle c|),
\end{aligned}
$$

where $|i\rangle\langle i|, i=l, c, r$, are projection operators for the three electronic states and the vibrational mode, frequency $\omega$, is operated on by $\hat{d}$. The tunneling elements between the dots depend exponentially on the displacement operator of the vibrational mode, $\hat{x}=\Delta x_{z p}\left(\hat{d}^{\dagger}+\hat{d}\right)$, where $\Delta x_{z p}$ is the zero-point position uncertainty of the oscillator. The oscillator position has an expectation value of zero when the oscillator is unperturbed. The tunneling amplitude and length are given by the positive quantities $V$ and $1 / \alpha$ respectively. Although we have not stated it explicitly, it is understood that the potential energy of the vibrational modes has a hard wall cut-off at the left and righthand dots (i.e., at $\left.x= \pm x_{0}\right)$.

The energies of the outer two dots, $\varepsilon_{l(r)}$, together define a voltage bias across the device, $e V_{b}=\varepsilon_{b}=\varepsilon_{l}-\varepsilon_{r}$. The energy of the central level is set by its position between the puter dots as we assume that it undergoes a Stark shift proportional to its position 18 so that $\varepsilon_{c}=\varepsilon_{l}-\left(\hat{x}+x_{0}\right) \varepsilon_{b} / 2 x_{0} 19$ The energy levels in the outer dots are set by external gates whose capacitance is assumed to be much larger than the capacitances of the other junctions (i.e. the junctions between the dots and the junctions between the outer dots and the leads), but still small enough that only one of the dots can be occupied by an electron at any one time.

The behavior of the shuttle system is readily investiagted by numerical methods. The Hamiltonian and density operators are represented as matrices, as described in appendix A, and the time evolution of the system can be obtained numerically for any initial form of the density matrix. However, the vibrational degree of freedom is only completely specified by an infinite set of states which must be truncated for numerical calculations. The necessary truncation is best performed in the basis of the (unperturbed) energy eigenstates of the vibrational mode (see appendix A). So long as the energy of the highest energy state included is much larger than any other energy scale in the problem, then the error due to truncation is small.

\section{B. Coupling to leads}

The transport properties of the shuttle system coupled to the leads are obtained by integrating an equation of motion for the density matrix appropriate to an open quantum system. Apart from the internal dynamics of the shuttle, there are two effects we need to take account of. Couplings between the electronic states in the outer dots and the leads must be incorporated, and the coupling between the oscillator and its environment must also be included.

The external couplings of the outer dots and oscillator are accounted for in the dynamics via additional terms in the equation of motion for the reduced density matrix of the shuttle, an approach which is well-kneym in the field of quantum optics and has recently been applied to problems of electron transport in nanostructures.16.20 The electrons in the leads are assumed to be completely incoherent which means that all the off-diagonal elements of the density matrix between the state $|0\rangle$ and the other electronic states can be set to zero (notice, however, that the diagonal element in the density matrix for $|0\rangle$ has both diagonal and off-diagonal matrix elements in the space of the vibrational mode states).

The appropriate equation of motion for the density matrix of the system generalised to include the leads and the environment of the oscillator therefore takes the general form

$$
\dot{\rho}=-\frac{i}{\hbar}[H, \rho]+\Xi \rho+\dot{\rho}_{d}
$$

where the 'decay matrix', $\Xi$, incorporates transitions between the leads and the outer dots and $\dot{\rho}_{d}$ accounts for the decohering and dissipative effects of the oscillator's environment. The form of the non-unitary terms can be derived by adapting standard techniques used in the field of quantum optics and we will consider them both in turn.21

The form of decay matrix coupling the shuttle to the leads can be obtained using the method of Gurvitz et al., 20 as described in appendix B. The components of the matrix, $[\Xi \rho]_{a b}^{i j}$, are labelled by the vibrational state (superscripts) and the electronic states (subscripts). The matrix is Hermitian and is specified by the components22

$$
\begin{aligned}
& {[\Xi \rho]_{l l}^{i j}=\rho_{00}^{i j} \Gamma} \\
& {[\Xi \rho]_{r r}^{i j}=-\rho_{r r}^{i j} \Gamma} \\
& {[\Xi \rho]_{00}^{i j}=-\Gamma\left(\rho_{00}^{i j}-\rho_{r r}^{i j}\right)}
\end{aligned}
$$




$$
\begin{aligned}
& {[\Xi \rho]_{r c}^{i j}=-\frac{\Gamma}{2} \rho_{r c}^{i j}} \\
& {[\Xi \rho]_{l r}^{i j}=-\frac{\Gamma}{2} \rho_{l r}^{i j}} \\
& {[\Xi \rho]_{c c}^{i j}=[\Xi \rho]_{l c}^{i j}=0,}
\end{aligned}
$$

where $\Gamma$ is the tunneling rate between the dots and the leads, and all terms $[\Xi \rho]_{0 a}^{i j}$ where $a \neq 0$ are zero as the associated elements of the density matrix $\left(\rho_{0 a}^{i j}\right.$ with $\left.a \neq 0\right)$ are zero by definition.

Including the environment of the vibrational mode is essential to the description as it is the dissipation arising from this coupling which gives rise to a steady-state in which the current is constant, as we discuss below. We employ a minimal model of the environment, assuming it to be composed of a bath of oscillators at a fixed temperature $T$, to which the vibrational mode is coupled only weakly Under these assumptions the dissipative component in the equation of motion for the density matrix, is given by 21

$$
\begin{aligned}
\dot{\rho}_{d}= & -\frac{\gamma}{2} \bar{n}\left(\hat{d} \hat{d}^{\dagger} \rho-2 \hat{d}^{\dagger} \rho \hat{d}+\rho \hat{d} \hat{d}^{\dagger}\right) \\
& -\frac{\gamma}{2}(\bar{n}+1)\left(\hat{d}^{\dagger} \hat{d} \rho-2 \hat{d} \rho \hat{d}^{\dagger}+\rho \hat{d}^{\dagger} \hat{d}\right),
\end{aligned}
$$

where $\gamma$ is the classical damping rate of the oscillator, and $\bar{n}$, is the usual thermal occupation number of the oscillator at temperature $T$,

$$
\bar{n}=\frac{1}{\mathrm{e}^{\hbar \omega / k_{\mathrm{B}} T}-1}
$$

The classical damping rate is just the rate at which the vibrational mode lpses energy due to frictional forces, its value can be obtained empirically from the quality factor of the oscillator $21=\omega / Q$.

The steady-state current through the system (in units of electrons per unit time) is given by

$$
\frac{I}{e}=\Gamma \rho_{r r}^{(s)},
$$

where $\rho_{r r}^{(s)}$ is the occupation probability of the righthand dot when a steady state has been achieved. In practice the current is determined after evolving the equation of motion for the density matrix until further changes with time become negligible.

The discrete nature of the states in the dots has an important effect on the ways in which energy can be transferred in the shuttle. An electron travelling through the shuttle must enter at an energy determined by the level in the lefthand dot and leave at the energy determined by the lower level in the righthand dot. Hence each electron travelling threjgh the device dissipates an amount of energy proportional to the bias voltage. For a system without an oscillator, 18 the energy is dissipated in the lead with the lower chemical potential. However, for the shuttle we consider here, some of the energy ends up in the oscillator - rather like the electron 'pump' considered by Stafford and Wingreen in reverse.23 Despite this apparent pumping mechanism for the oscillator, the degree to which the oscillafor can be excited is strongly limited by damping and a steady-state is always achieved when this effect is included.26

\section{EIGENVALUE DYNAMICS OF THE ISOLATED SHUTTLE}

For a system in which the coupling to external leads and the environment 18 is much weaker than the coupling between the dots (i.e. when $\Gamma, \gamma \ll V \mathrm{e}^{-\alpha x_{0}} / \hbar$ ), the current characteristics are expected to be strongly influenced by the eigenstates of the isolated system. Under these conditions, we can think of the eigenstates of the system, which in general are not localised on any one dot, as forming independent channels for conduction. The current is carried most effectively by those eigenstates in which there is a finite probability of finding an electron in both the left and righthand dots, and the characteristics of the eigenstates can be determined from the behavior of the corresponding eigenvalues. Therefore we examine dynamics of the eigenvalues as a function of the bias voltage in the uncoupled shuttle system before going on to consider the corresponding behavior of the current in the coupled system.

The eigenvalue spectrum of the shuttle at zero bias voltage is controlled by the relative values of the energy scales of the oscillator, $\hbar \omega$, and the tunneling matrix element, $V$; and by the length-scales $x_{0}$ and $1 / \alpha$. The eigenvalue dynamics in the limit where the dots are uncoupled (i.e. $V=0$ ) is illustrated in Fig. 2a [note that all the energies in the figures are measured in units of $\hbar \omega$, and the distances are measured in units of $\left.\Delta x_{z p}\right]$. The energy levels at zero-bias are three-fold degenerate states separated by $\simeq \hbar \omega$. For small applied bias, the splittings between energy 
levels are linear. The spacing between the sets of three levels at zero-bias are not exactly $\hbar \omega$, but increase slightly with energy. This is because the oscillator is in fact confined by hard wall potentials which it begins to feel at higher energies, leading to an increase in the eigenenergies.

When the electronic states are decoupled the individual dot states are readily identified: the left and righthand dot energy levels increase and decrease respectively with increasing voltage bias, whilst the energy of the central-dot electronic states drop off quadratically (this state is equivalent to an oscillator in a linear potential). The eigenvalues for the left and righthand energy levels cross for $\varepsilon_{b} \simeq n \hbar \omega$ with $n$ an integer. For $\varepsilon_{b} \simeq 2 \hbar \omega$, the crossing of the left and righthand-dot energy levels almost coincides with the central-dot energy level. The level crossings of the lower eigenvalues occur at almost precisely integer values of $\hbar \omega$, but at higher energies the level crossings drift to larger bias values because of the increased spacing between levels at zero-bias.

For finite values of the tunneling matrix element, $V$, the eigenvalue dynamics as a function of $\varepsilon_{b}$ becomes more complex, as shown in Fig. 2. The energy levels at zero-bias split so that the degeneracy of the levels is lifted. At finite bias the levels no longer cross, but instead repel each other. The strength of the level repulsion depends strongly on $V$, but the oscillator state also plays a role as larger number states for the oscillator lead to stronger couplings between levels. However, away from the anti-crossings, the levels vary with $\varepsilon_{b}$ in almost the same way as for the decoupled case, implying that the eigenvalues in these regions can still be associated with states localised on the individual dots.

The level repulsions which occur at $\varepsilon_{b} \simeq n \hbar \omega$ for finite $V$ have a different character for odd and even values of $n$. For odd values of $n$, levels associated with the left and righthand dots anti-cross, leading to mixing of the levels. For even values of $n$, levels associated with the central dot mix first with levels associated with the lefthand dot and then with levels associated with the righthand dot within a very narrow range of $\varepsilon_{b}$. The simultaneous curvature of the three levels implies that, over a narrow range of $\varepsilon_{b}$, the mixing involves all of them. The lefthand dot level mixes with the central dot level first partly because the central dot level curves downwards, and partly because the lefthand dot level is associated with a less excited state of the oscillator which lies closer to the central dot level at zero-bias than the righthand dot level.

In the vicinity of the level anti-crossings states associated with either the lefthand and righthand dots, or with all three dots, become strongly mixed. The mixing of these levels implies that the electron will be highly delocalised near the anti-crossings, leading to a strong enhancement of the current through the shuttle. The variation in the location and strength of anti-crossings of levels (and hence mixings of states associated with different dots) with the eigenvalue energy implies that the actual current characteristics will be sensitive to the energy of the shuttle in the steady state.

At zero bias the system will be at resonance as the dot energy levels will be aligned, and we expect a large current to flow. However, the large current at zero bias can also be understood in terms of the current channels formed by the eigenstates. At very small bias energies the eigenvalues for finite $V$ have a quadratic dispersion, and the eigenstates are composed of almost symmetrical mixtures of the lefthand, central and righthand dot states and hence are highly delocalised.27

In studying the eigenspectra and the steady-state current we concentrate on a range of bias values of a few $\hbar \omega$, for a combination of reasons. As the bias voltage increases, the eigenspectra become more complex. The spread in the locations of the anti-crossings with eigenenergy increases with $\varepsilon_{b}$ and the energies of the levels which anti-cross increase. The complexity of the eigenspectra at large values of $\varepsilon_{b}$ means that it will become increasingly difficult to understand the current characteristics of the system by reference to the eigenspectrum. Furthermore, our simple modelling of the electronic part of the system, including only a single energy level for each dot, will become increasingly unreliable as the bias is increased and higher levels in the dots become accessible.

\section{CURRENT CHARACTERISTICS}

In order to obtain the current characteristics of the shuttle system we integrate the equation of motion for the density matrix from a given initial state, until a steady state is obtained. The steady state is achieved when the energy gained by the oscillator from the electrons is matched by losses due to damping by the surrounding medium

The steady-state current for a chain of quantum dots at fixed positions has been studied extensively. $17.28,24.25,28$ In the Coulomb blockade regime, it is found that if the energy level in each of the dots is shifted by an amount proportional to its position (forming a 'Stark ladder'), the decay in current with the shift in energy levels is rapid. 18 For the shuttle, we find that the presence of the oscillator leads to significant changes in the current characteristics.

We begin by analysing the simplest case, where the dots are strongly coupled to each other (and weakly coupled to the leads) and the temperature is set to zero. In this regime the current characteristics can be understood in terms of the energy eigenspectrum of the system and the effect of the oscillator's environment which causes scattering between the eigenstates. However, we also consider the behavior when the tunneling length of the electrons is reduced, so that the coupling between the dots becomes weak, as it is in this regime that current can flow hy hopping onto then off the central dot sequentially, in a manner very reminiscent of the semi-classical shuttles 10 . 6 - Furthermore, in practice, 
temperature will play an important role and so we extend our analysis to small but finite temperatures. Working at finite temperatures complicates the numerical calculation as it naturally increases the importance of higher energy oscillator states. This means that care needs to be taken in truncating the matrix of oscillator states (as described in appendix A) to ensure that the results obtained do not depend strongly on the size of the truncated Hamiltonian matrix. The errors due to truncation only become significant for temperatures such that $\hbar \omega \ll k_{\mathrm{B}} T$. The matrix method can readily be adapted to investigate the effect of temperature when $\hbar \omega \sim k_{\mathrm{B}} T$.

The initial state of the system describes the initial wavefunctions of the electronic and oscillator parts of the system. The initial form of electronic part of the wavefunction is unimportant: it simply describes the initial probabilities of finding an electron on each of the dots, which does not in practice affect the form of the steady state which is later achieved. However, the initial state of the oscillator is of some importance as it depends on the background temperature, but at zero temperature, the oscillator is initially in its ground state.

The steady-state current through the dots as a function of $\varepsilon_{b}$ with and without the oscillator is illustrated in Fig. 3 for the case of strong coupling between the dots. The tunneling rate to the leads is $\Gamma=0.05$ and the damping rate for the quantum oscillator is $\gamma=0.025$ [all rates are measured in units of $\omega$ ].

When the bias is zero, all three dot levels are aligned leading to resonant transmission of electrons. Without the oscillator, the current decays rapidly with increasing bias, $\varepsilon_{b}$, as expected. When the oscillator is present, the current initially decays with increasing bias, though more slowly than before, but at larger values of $\varepsilon_{b}$ prominent current resonances dominate the behavior.

For strong coupling between the dots (compared to the couplings to the leads), the current peaks are best described in terms of the conduction channels formed by the eigenstates of the shuttle system. Comparison of the current peaks in Fig. 3 with the corresponding eigenspectrum in Fig. 2 shows that the resonances coincide with avoided-level crossings where the eigenstates are formed from mixtures of states localised on the individual dots. Further resonances should occur at higher values of $\varepsilon_{b}$, but the model will eventually break down when the shift in levels exceeds the energy level spacing in the dots themselves. The peak in the current at zero bias arises because the eigenstates for $\varepsilon_{b}=0$ each consist of (symmetrical) mixtures of the states associated with each of the dots. Of course the peak at zero bias can also be seen as due to resonant tunneling through the shuttle.

The second current peak, which occurs at $\varepsilon_{b} \simeq 0.86$ (in units of $\hbar \omega$ ), is due to the mixing of levels associated with the left and righthand dots. The resulting eigenstates are symmetrical as they contain almost equal weights for the left and righthand dots (i.e. in these states the probability of finding the electron on the left and righthand dots are are almost equal). The third current peak occurs at $\varepsilon_{b} \simeq 1.95$, and corresponds to a mixing of the central dot level, more or less simultaneously, with both left and righthand dot levels. In this case the resulting eigenstates are not entirely symmetrical: there are larger weights for the lefthand dot in some states and larger weights for the righthand dots in others. The fourth current peak, occuring at $\varepsilon_{b} \simeq 2.92$, is similar to the second peak, in that it too is due to the mixing of levels associated with the left and righthand dots. The magnitude of the fourth current peak is rather lower than that of the second peak, partly because the left and righthand dot levels which are mixed (at $\left.\varepsilon_{b} \simeq 2.92\right)$, correspond to oscillator states differing by three quanta (whereas states differing by only one oscillator quantum are mixed at $\varepsilon_{b} \simeq 0.86$ ), and partly because anti-crossings progressively disappear from the lower part of the energy spectrum as the bias is increased.

\section{A. Effects of oscillator damping}

The dynamics of the system is strongly influenced by the magnitude of the oscillator damping, $\gamma$. Without any damping, the oscillator would gain energy continuously from the electrons and no steady state would be achieved (without including higher electronic levels). The effect of $\gamma$ in producing a steady state can be seen by examining the average energy of the shuttle as a function of time.

The average energy is readily computed from the density matrix and Hamiltonian of the system (suitably generalised to account for the energy of the oscillator when none of the dots are occupied). Fig. 1 shows the evolution of the average energy, $\langle E\rangle=\operatorname{Tr}[H \rho]$, as a function of time for a range of different damping constants. The average energy at $t=0$ is given by the initial state of the system with the oscillator in its ground state and an electron present on the lefthand dot (the bias energy, $\varepsilon_{b}=0.86$, corresponds to a peak in the current). For each value of $\gamma$, the average energy varies with time before eventually settling down to a constant value, indicating that the system has reached a steady-state.

The time taken to reach the steady-state, and the average energy in that state vary considerably. For $\gamma \sim 0.025$ the oscillator appears to be 'critically damped' in the sense that the energy in the steady-state is roughly the initial energy of the system. In contrast, for $\gamma>0.025$ the system achieves a steady state by losing energy to the environment, implying over-damping of the oscillator. As soon as the electron leaves the lefthand dot and moves to states on the other dots at lower energies, some of the initial energy is dissipated into the environment via the oscillator. When 
$\gamma<0.025$, the oscillator is under-damped and the system gains energy from the electron reservoirs as successive electrons pass through the system until a steady state is eventually achieved. For the underdamped case, the time taken to achieve the steady-state is longer than for either critical or over-damping and it eventually diverges when $\gamma$ is set to zero.

The value of the oscillator damping also has a strong effect on the magnitude of the steady-state current through the shuttle, as shown in Fig. 5. The way in which $\gamma$ affects the current depends sensitively on the value of $\varepsilon_{b}$. The first and second current peaks are almost unaffected by the variation in the oscillator damping constant, $\gamma$, whilst damping of the oscillator increases the current at the third peak and decreases it at the fourth peak. The reason for this differing behavior is readily understood in terms of a picture of independent conduction channels arising from the eigenstates of the shuttle. If the damping is weak, it can be thought of as causing scattering between channels with slightly differing energies. Therefore, damping can increase the current wherever scattering can transfer electrons from channels with a larger weights on the lefthand dot (where the electrons enter the system) to ones with large weights on the righthand dot (from which the electron leaves the system). At the first and second current peaks the states which carry the current are largely symmetrical, in that they contain almost equal weights for the left and righthand dots, so the damping cannot increase the current. In contrast, at the third peak the current can increase as there is considerable asymmetry between nearby eigenstates. At the fourth current peak, increasing the damping reduces the current because at this bias energy the current is very sensitive to the reduction in the average energy caused by the higher damping.

\section{B. Current in weak coupling regime}

At larger values of $\alpha$ (i.e. for smaller tunneling lengths $\lambda=1 / \alpha$ ) the coupling between the dots grows weaker and the picture of the current as arising from the passage of electrons through weakly coupled eigenstates acting as conduction channels begins to break down. Instead we must think of the electrons as tunneling between states localised on the individual dots.

When the bias energy is $\sim \hbar \omega$ or $3 \hbar \omega$ the electrons must tunnel coherently from the lefthand dot to the righthand dot via a virtual (non-energy conserving) transition to the central dot. However, when the bias energy is $\sim 2 \hbar \omega$ electrons can tunnel through the system incoherently via two separate tunneling events in each of which a quanta of energy is dumped in the oscillator. Of course when $\varepsilon_{b}=0$ there is no need to dump energy into the oscillator and all tunneling processes through the central dot conserve energy.

The effect of increasing $\alpha$ is shown clearly in Fig. 6: the second and fourth peaks are strongly suppressed, whilst the third peak is only slightly reduced. As the tunneling length becomes smaller, coherent tunneling directly between the left and righthand dots becomes more difficult. However, electrons can still flow through the device by tunneling onto the central dot and then off again later in a sequence of two separate tunneling events, i.e. current flows by shuttling of electrons through the central dot.

As for strongly coupled dots, an increase in damping of the oscillator enhances the current when $\varepsilon_{b} \simeq 2 \hbar \omega$. When the coupling between the dots is weak the damping assists current flow by removing energy from the oscillator and thereby helps prevent electrons from tunneling backwards (i.e from the righthand dot to the central dot or from the central dot to the righthand dot).

\section{Finite temperatures}

The effects of finite temperature on the current characteristics of the shuttle can be taken into account by generalising the initial state of the oscillator to a thermal mixture and modifying the damping terms (described in section II) to take account of the environment's temperature. As long as the temperature does not approach the charging energies associated with the Coulomb blockade on the dots, no adjustment to the electronic part of the model is required.

Fig. 7 compares the current through the shuttle for a particular choice of parameters at $k_{\mathrm{B}} T=0$ and $k_{\mathrm{B}} T=3$ (in units of $\hbar \omega)$. The finite temperature has two noticeable effects. Firstly, the current characteristics are smeared out: the peaks are broader and lower, while the troughs are shallower. Secondly, the maxima of the peaks are shifted to slightly higher bias energies.

The effect of finite temperature on the current is readily interpreted with reference to the eigenspectra in Fig. 2. The finite temperature increases the average energy of the system, whilst also broadening the distribution of probabilities of finding the system in a given state. The broadening of the probability distribution implies that a whole range of states contribute to the current, each of which have anti-crossings occuring at different values of $\varepsilon_{b}$, leading to a broadening of the current peaks. The overall increase in the average energy leads to a general increase in 
the current because of the increased strength in the anti-crossings at higher energies, especially at larger values of $\varepsilon_{b}$ where anti-crossings no longer occur in the lower parts of the eigenspectrum.

\section{CONCLUSIONS AND DISCUSSION}

We have investigated the effect of a quantised vibrational degree of freedom on transport through a chain of three quantum dots where the tunneling between the dots depends exponentially on the displacement of the oscillator. When the dots are strongly coupled to each other and weakly coupled to the leads, the current through the chain can be predicted by looking at where anti-crossings in the eigenspectrum give rise to electronic states which are delocalised amongst the dots.

The damping of the oscillator due to coupling to its environment has important effects on the current flowing through the system. The current through the shuttle only reaches a steady value when the energy pumped into it from the electrons is balanced by the dissipation due to the oscillator's environment. For weak damping, the environment can be thought of as causing scattering between different current channels. The scattering enhances the current whenever it can transfer electrons from states strongly concentrated on the lefthand dot to ones strongly concentrated on the righthand dot. However, when the eigenstates contain equal probabilities of finding the electron on the lefthand and righthand dots, scattering has no effect on the current.

When the tunneling length is reduced the effective coupling between the dots is also reduced and the picture in terms of independent conduction channels can no longer be applied. Instead we can think of the electrons as passing through the shuttle via transitions between states localised on each of the dots. When the energy difference is an odd number of vibrational quanta, current flow is via a virtual transition. In contrast, when the energy difference is an even number of vibrational quanta, electrons can tunnel sequentially onto and then off the central dot. The virtual transitions are strongly suppressed by reductions in the tunneling length of the electron. However, the sequential transitions - electrons hopping on and then off the central dot-are much less affected by changes in the tunneling length.

The transport of eleftrons through the central dot via sequential tunneling, is analogous to the semi-classical models of electron-shuttling. 3 As in the case of the experiments of Blick et al.5.6 the vibrational mode is driven and current flows by electrons tunneling onto the central dot and then tunneling off in the opposite direction at a later time. However, for the system we consider the driving energy comes from the electrons themselves as the difference in dot energy levels forces them to lose energy to the oscillator.

The shuttling mechanism dominates over higher order processes when the tunneling length, $\lambda$, is of order the zeropoint position uncertainty of the oscillator, $\Delta x_{z p}$, and can give rise to a larger current than at the electronic resonance (i.e. when $\varepsilon_{b}=0$ ). However, the sharp features in the current characteristics begin to be smeared out when $k_{\mathrm{B}} T>\hbar \omega$.

In order to have $k_{\mathrm{B}}$ Tsim $\hbar \omega$ in the range $0.1-1 \mathrm{~K}$ the frequency of the oscillator must be $\sim 1-10 \mathrm{GHz}$. This high frequency range is accessible in a wide variety of systems: the elastic links in nanopartice arrays have frequencies 14 $\sim 10 \mathrm{GHz}$, micromechanical resonators can be fabricated with frequencies approaching $1 \mathrm{GHz}, 29$ and indeed the vibrational modes in many molecular systems have much higher frequencies. 8 The tunneling length depends on the work function of the surfaces involved, but typically lies in the range $30.05-3 \AA$. Unfortunately, raising the frequency of the oscillator reduces the zero-point position uncertainty, $\Delta x_{z p}=\sqrt{\hbar / 2 m \omega}$, but the effective-masses of even artifically fabricated oscillators can be extremely small (for example nanoparticles can have masses $\mathbb{1 4}^{14} \sim 10^{-23} \mathrm{~kg}$ ). Therefore, it should be possible to fabricate or assemble systems in which both $\lambda \sim \Delta x_{z p}$ and $k_{\mathrm{B}} T \sim \hbar \omega$, and thereby observe electron shuttling in the quantum regime.

One of us (A.M.) would like to thank The Cavendish Laboratory, at the University of Cambridge, for their hospitality. This work was funded by the EPSRC under grant GR/M42909/01.

\section{APPENDIX A: HAMILTONIAN IN MATRIX FORM}

The Hamiltonian of the isolated system of three dots and an oscillator has three electronic states and a number of vibrational states which is in principle infinite, but which in practice we limit to a number $N \sim 25$ so that the largest energy state of the oscillator $\left(E_{\max }=N \hbar \omega\right)$ is much greater than any other energy scale in the problem.

The full Hamiltonian for the shuttle consists of nine $N \times N$ sub-matricies, corresponding to the three electronic states and the tunneling elements between them

$$
H=\left(\begin{array}{ccc}
H_{L L} & H_{L C} & H_{L R} \\
H_{C L} & H_{C C} & H_{C R} \\
H_{R L} & H_{R C} & H_{R R}
\end{array}\right)
$$


The sub-matrices have rows and columns labelled in a suitable basis of the the oscillator states. In the number representation of the oscillator states the diagonal sub-matrices take the form

$$
\begin{aligned}
\left\langle n\left|H_{L L}\right| m\right\rangle= & \left(\varepsilon_{l}+m \hbar \omega\right) \delta_{n m} \\
\left\langle n\left|H_{C C}\right| m\right\rangle= & \frac{\varepsilon_{d}}{\Delta x_{z p}}\left\langle n\left|\left(\hat{x}+x_{0}\right)\right| m\right\rangle \\
& +\left(\varepsilon_{l}+m \hbar \omega\right) \delta_{n m} \\
\left\langle n\left|H_{R R}\right| m\right\rangle= & \left(\varepsilon_{r}+m \hbar \omega\right) \delta_{n m},
\end{aligned}
$$

where $\varepsilon_{d}=\Delta x_{z p}\left(\varepsilon_{r}-\varepsilon_{l}\right) / 2 x_{0}$, with $\Delta x_{z p}$ the zero-point position uncertainty of the oscillator. Of the other submatrices, only those coupling adjacent dots (i.e. $H_{L C}$ and $H_{C R}$, and their Hermitian conjugates) are non-zero,

$$
\begin{aligned}
& \left\langle n\left|H_{L C}\right| m\right\rangle=-V\left\langle n\left|\mathrm{e}^{-\alpha\left[x_{0}+\hat{x}\right]}\right| m\right\rangle \\
& \left\langle n\left|H_{C R}\right| m\right\rangle=-V\left\langle n\left|\mathrm{e}^{-\alpha\left[x_{0}-\hat{x}\right]}\right| m\right\rangle .
\end{aligned}
$$

The most efficient representation of the oscillator states for computational purposes is the position basis. The oscillator displacement is restricted to lie between the values set by the positions of the outer dots, a restriction which is readily enforced in the position representation. The set of position states is obtained by diagonalising the position operator in the number basis (a matrix of size $N \times N$ ), then those states amongst the resulting $N$ position states with eigenvalues outside the range $\left(-x_{0}, x_{0}\right)$ are projected out, leading to a somewhat smaller sub-matrix. This simple-minded procedure is appropriate at relatively low energies when the weight of the wavefunction in the region outside the cut-offs is relatively small.

\section{APPENDIX B: DENSITY MATRIX EQUATION OF MOTION}

In this appendix we detail how the dissipative terms in the density matrix equation of motion are obtained when the voltage difference between the leads is less than the spacing of energy levels within each of the dots, so that transport is restricted to the Coulomb blockade regime. We begin by deriving the rates which describe the transitions to and from the leads which are attached to the system, before going on to calculate the equation of motion for the density matrix following the prescription of Gurvitz et al.20

Following the usual theory of single electron tunneling 3 we can write the net tunneling rate from a lead with chemical potential $\mu$ through a barrier to an isolated state with effective energy $\varepsilon$ as

$$
\Gamma_{n e t}=V_{0}[P(0) f(\Delta)-P(1)(1-f(\Delta))]
$$

where $\Delta=\varepsilon-\mu, V_{0}$ is the tunneling amplitude which is assumed to be independent of the energy, $f$ is the Fermi distribution function and $P(0)(P(1))$ is the probability that the isolated state is unoccupied(occupied).

For the shuttle, we need to consider transitions between the lefthand lead and lefthand dot, and between the righthand lead and righthand dot. In addition, the strong Coulomb blockade means that transitions onto any of the dots can only occur if all of the dots are unoccupied. Therefore, for transitions between the lefthand lead and left-hand dot, $P(0)=\rho_{0}$ where we use the notation $\rho_{k}=\sum_{i} \rho_{k k}^{i i}$, for the probability that the electronic state $k$ is occupied, with the superscripts and subscripts of the density matrix labelling the oscillator and electronic states respectively. Thus the net tunneling rate from the left-hand lead to the left-hand dot takes the form

$$
\Gamma_{n e t}^{L}=\Gamma_{L}^{+} \rho_{0}-\Gamma_{L}^{-} \rho_{l}
$$

where $\Gamma_{L}^{+}=V_{0} f(\Delta), \Gamma_{L}^{-}=V_{0}(1-f(\Delta))$. Similarly, we find

$$
\Gamma_{n e t}^{R}=\Gamma_{R}^{+} \rho_{r}-\Gamma_{R}^{-} \rho_{0} .
$$

These net rates can be separated into individual terms which enter into the equations of motion of the elements of the density matrix. We assume that $\Delta=\varepsilon_{l}-\mu_{l}=\mu_{r}-\varepsilon_{r}$, where $\mu_{l(r)}$ is the Fermi level in the left(right) lead. The tunneling rates for the righthand lead take the form $\Gamma_{R}^{-}=V_{0} f(-\Delta)$ and $\Gamma_{R}^{+}=V_{0}(1-f(-\Delta))$.

In order to simplify the analysis, we will work in the large bias limit where $|\Delta| \gg k_{\mathrm{B}} T$. In this limit, the backward tunneling rates $\Gamma_{L(R)}^{-}$go to zero and the forward rates take the simplified form $\Gamma_{L(R)}^{+}=V_{0}$. We shall restrict our analysis to this regime for simplicity, but the generalisation to the master equation for the case where $|\Delta| \sim k_{\mathrm{B}} T$ is relatively straightforward. 
Gurvitz et al. 20 have developed a way of extending the density matrix description of electronic nanostructures to include couplings between localised, coherent, electronic states and leads containing a very large number of states where the electrons can be regarded as incoherent. According to this approach, the equation of motion for the density matrix including couplings to the leads (but not the environment of the oscillator) takes the general form 31 (setting $\hbar=1)$

$$
\begin{aligned}
& \dot{\rho}_{a a}^{i j}=-i[H, \rho]_{a a}^{i j}-\rho_{a a}^{i j} \sum_{d \neq a} \Gamma_{a \rightarrow d}+\rho_{c c}^{i j} \sum_{c \neq a} \Gamma_{c \rightarrow a} \\
& \dot{\rho}_{a b}^{i j}=-i[H, \rho]_{a b}^{i j}-\frac{\rho_{a b}^{i j}}{2}\left(\sum_{d \neq a} \Gamma_{a \rightarrow d}+\sum_{d \neq b} \Gamma_{b \rightarrow d}\right)
\end{aligned}
$$

where the superscripts on the density matrix label the vibrational state and the subscripts label the electronic parts. The terms $\Gamma_{n \rightarrow m}$ give the transition rates from states $|n\rangle$ to $|m\rangle$.

Following the prescription given in Eqn.s (B4) and (B5), and taking into account only tunneling onto the left-hand dot from the left-hand lead and tunneling off the righthand dot into the righthand lead, we obtain

$$
\begin{aligned}
& \dot{\rho}_{l l}^{i j}=-i[H, \rho]_{l l}^{i j}+\rho_{0}^{i j} \Gamma_{L} \\
& \dot{\rho}_{c c}^{i j}=-i[H, \rho]_{c c}^{i j} \\
& \dot{\rho}_{r r}^{i j}=-i[H, \rho]_{r r}^{i j}-\Gamma_{R} \rho_{r r}^{i j} \\
& \dot{\rho}_{0}^{i j}=-i[H, \rho]_{00}^{i j}-\Gamma_{L} \rho_{0}^{i j}+\Gamma_{R} \rho_{r r}^{i j} \\
& \dot{\rho}_{l c}^{i j}=-i[H, \rho]_{l c}^{i j} \\
& \dot{\rho}_{l r}^{i j}=-i[H, \rho]_{l r}^{i j}-\frac{1}{2} \Gamma_{R} \rho_{l r}^{i j} \\
& \dot{\rho}_{c r}^{i j}=-i[H, \rho]_{c r}^{i j}-\frac{1}{2} \Gamma_{R} \rho_{c r}^{i j},
\end{aligned}
$$

where we have omitted the superscript ' + ' from the tunneling rates and the terms off-diagonal in the electronic state $|0\rangle$ are zero, by definition, and so are omitted. Assuming the dot-lead junctions are identical, we can simplify the equations further by writing $\Gamma=\Gamma_{R}=\Gamma_{L}$, and the terms coupling the dots to the leads are given by the matrix, $\Xi \rho$, given in Eqn.s 3 8.

1 M.F. Bocko, K.A. Stephenson and R.H. Koch, Phys. Rev. Lett. 61, 726 (1988); B. Yurke and G.P. Kochanski, Phys. Rev. B 41, 8184 (1990)

2 N.F. Schwabe, A.N. Cleland, M.C. Cross and M.L. Roukes, Phys. Rev. B 52, 12911 (1995)

3 L.Y. Gorelik, A. Isacsson, M.V. Voinova, R. Kaesmo, R.I. Shekhter and M. Jonson, Phys. Rev. Lett. 80, 4526 (1998); A. Isacsson, L.Y. Gorelik, M.V. Voinova, R. Kaesmo, R.I. Shekhter and M. Jonson, Physica B 255, 150 (1998)

${ }^{4}$ M.T. Tuominen, R.V. Krotkov and M.L. Breuer, Phys. Rev. Lett. 83, 3025 (1999)

${ }^{5}$ R.H. Blick, A. Erbe, H. Kömmer, A. Kraus and J.P. Kotthaus, Physica E 6, 821 (2000)

6 A. Erbe, C. Weiss, W. Zwerger and R.H. Blick, Phys. Rev. Lett. 87, 096106 (2001)

7 C. Weiss and W. Zwerger, Europhys. Lett. 47, 97 (1999)

8 H. Park, J. Park, A.K. Lim, E.H. Anderson, A.P. Alivisotos and P.L. McEuen, Nature 407, 57 (2000)

9 S.H.M. Persson and L. Olofsson, Appl. Phys. Lett. 74, 2546 (1996)

10 D. Boese and H. Schoeller, Europhys. Lett. 54, 668 (2001)

11 N. Nishiguchi, Phys. Rev. B 65, 035403 (2001)

12 C. Jaochim, J.K. Gimzewski and A. Aviram, Nature 408, 541 (2000)

13 J.Gaudioso, L.J. Lauhon and W. Ho, Phys. Rev. Lett. 85, 1918 (2000)

14 N. Nishiguchi, Jpn. J. App. Phys. 40, 1 (2001)

15 A.D. Armour and A. MacKinnon, Physica B 316, 403 (2002)

16 T.H. Stoof and Y.V. Nazarov, Phys. Rev. B 53, 1050 (1996)

17 F. Renzoni and T. Brandes, Phys. Rev. B 64, 245301 (2001)

18 M.R. Wegewijs and Y.V. Nazarov, Phys. Rev. B 60, 14318 (1999)

19 The energy level in the central dot will in fact shift by a fraction of this amount which depend on the details of the inter-dot capacitances. However, we have neglected this detail in the interests of simplicity.

20 S.A. Gurvitz, H.J. Lipkin and Y.S. Prager, Phys. Lett. A 212, 91 (1996); S.A. Gurvitz, and Y.S. Prager, Phys. Rev. B 53, 15932 (1996) 
${ }^{21}$ M.O. Scully and M.S. Zubairy, Quantum Optics (Cambridge University Press, Cambridge U.K., 1997)

22 Explicitly, this matrix consists of a set of sub-matrices labelled by the electronic states, with the elements of each sub-matrix labelled by the oscillator states, just like the Hamiltonian described in appendix A

23 C.A. Stafford and N.S. Wingreen, Phys. Rev. Lett. 76, 1916 (1996)

24 P. Pals and A. MacKinnon, J. Phys. Cond. Matt. 8, 3177 (1996)

${ }^{25}$ P. Pals and A. MacKinnon, J. Phys. Cond. Matt. 8, 5401 (1996)

26 The level spacing in the dots also provides a fundamental limit on the excitation of the oscillator. When the excess energy in the oscillator approaches the electron spacing of the electrons in the dots it will be able to return energy to the electronic degrees of freedom. However, for simplicity we assume here that the oscillator energy is constrained entirely by damping.

27 We refer to the eigenstates as symmetrical in the sense that the probabilities of finding the electron on the left-hand and right-hand dots are equal.

28 C.A. Stafford, R. Kotlyar and S. Das Sarma, Phys. Rev. B 58, 7091 (1998)

29 A.N. Cleland and M.L. Roukes, in Proceedings of ICPS-24, edited by D. Gershoi (World Scientific, Singapore, 1999)

30 C.W.J. Beenakker, Phys. Rev. B 44, 1646 (1991)

31 In general, the formalism of Gurvitz et al. allows for an additional term in the equation of motion of the off-diagonal elements of the density matrix, known as coherence transfer terms. However, since these terms do not play a role in the system we consider here they have been omitted from the equation of motion. 


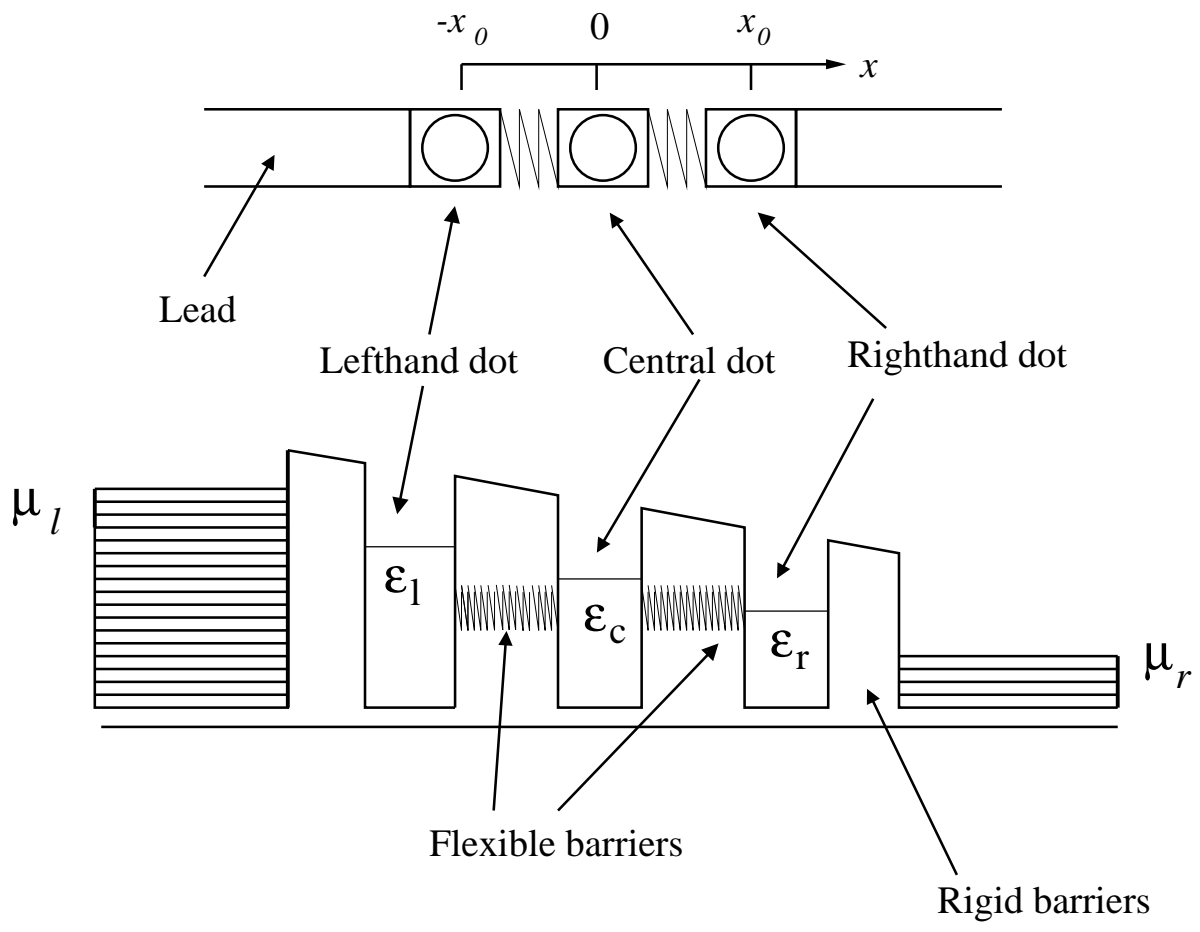

FIG. 1: Schematic diagrams of the shuttle system. The geometry and band structure of the system are shown in the upper and lower panels respectively. 

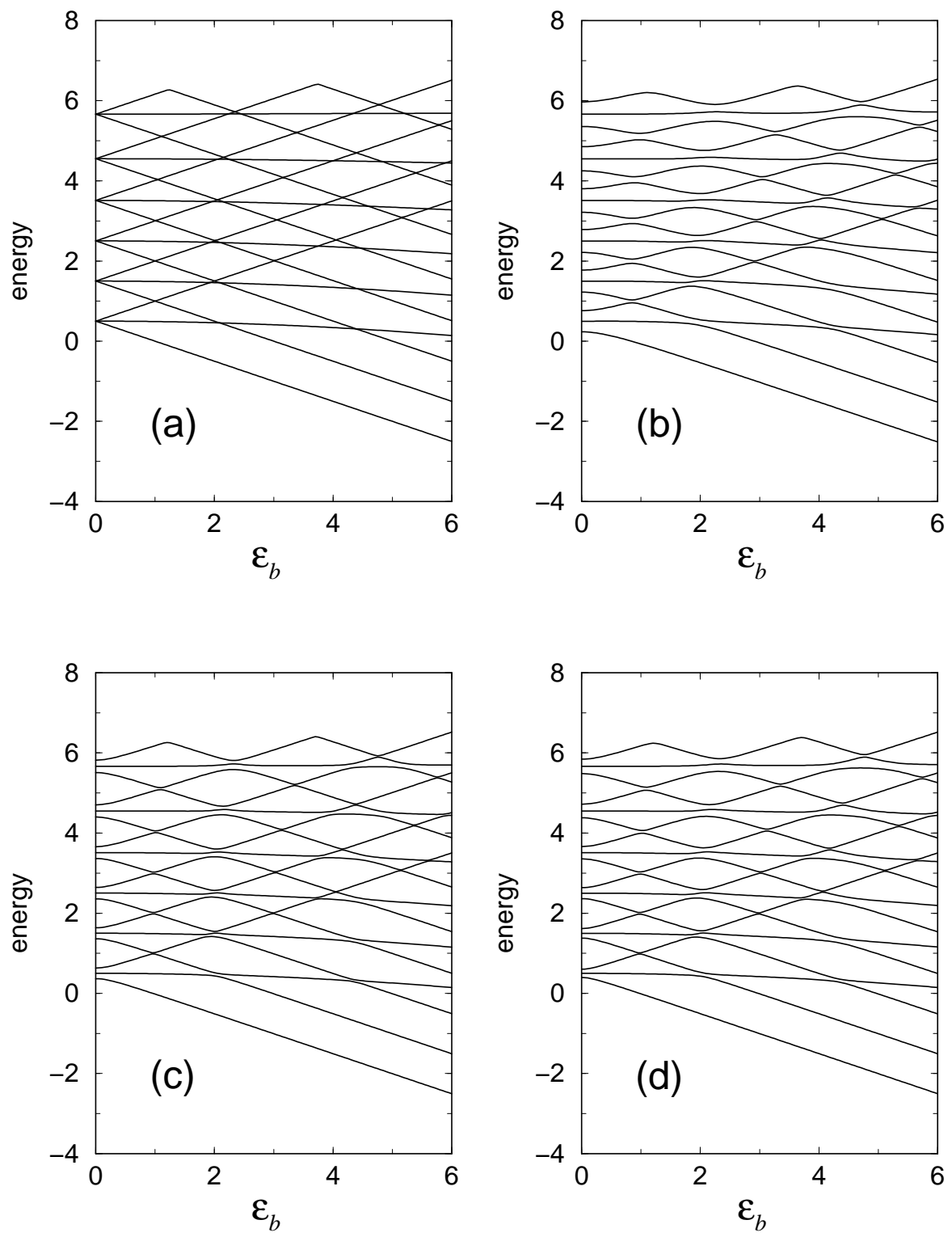

FIG. 2: Dynamics of the lowest 18 eigenvalues for systems with various parameter values. In the upper panels, $\alpha=0.2, x_{0}=5$ with $V=0$ (a) and $V=0.5(\mathrm{~b})$; in the lower panels $V=0.25, \alpha=0.2$ (c) and $V=0.5, \alpha=0.4$ (d). 


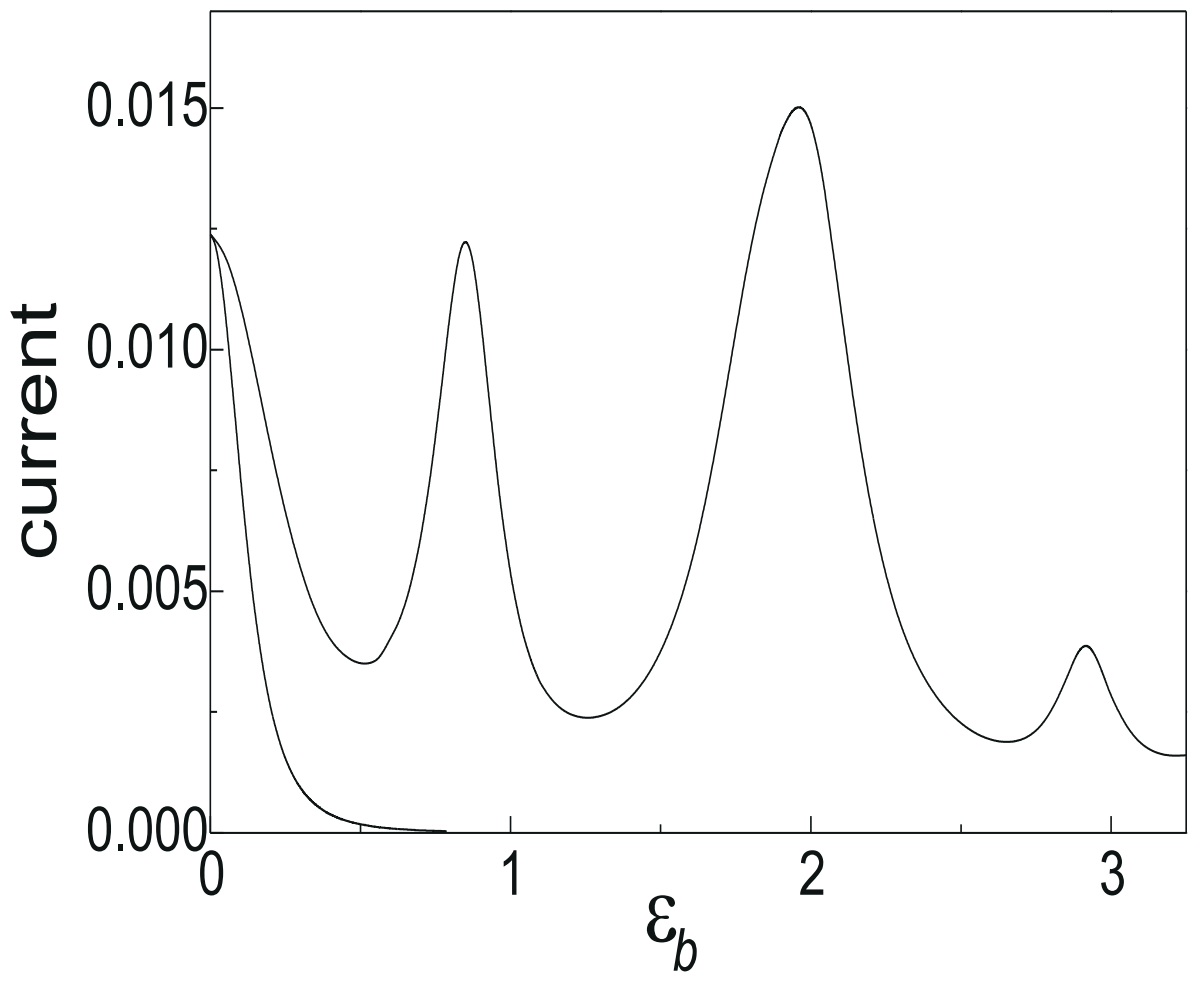

FIG. 3: Steady-state current through the shuttle with (upper curve) and without (lower curve) the oscillator; $V=0.5, \Gamma=0.05$, $\gamma=0.025, x_{0}=5$ and $\alpha=0.2$.

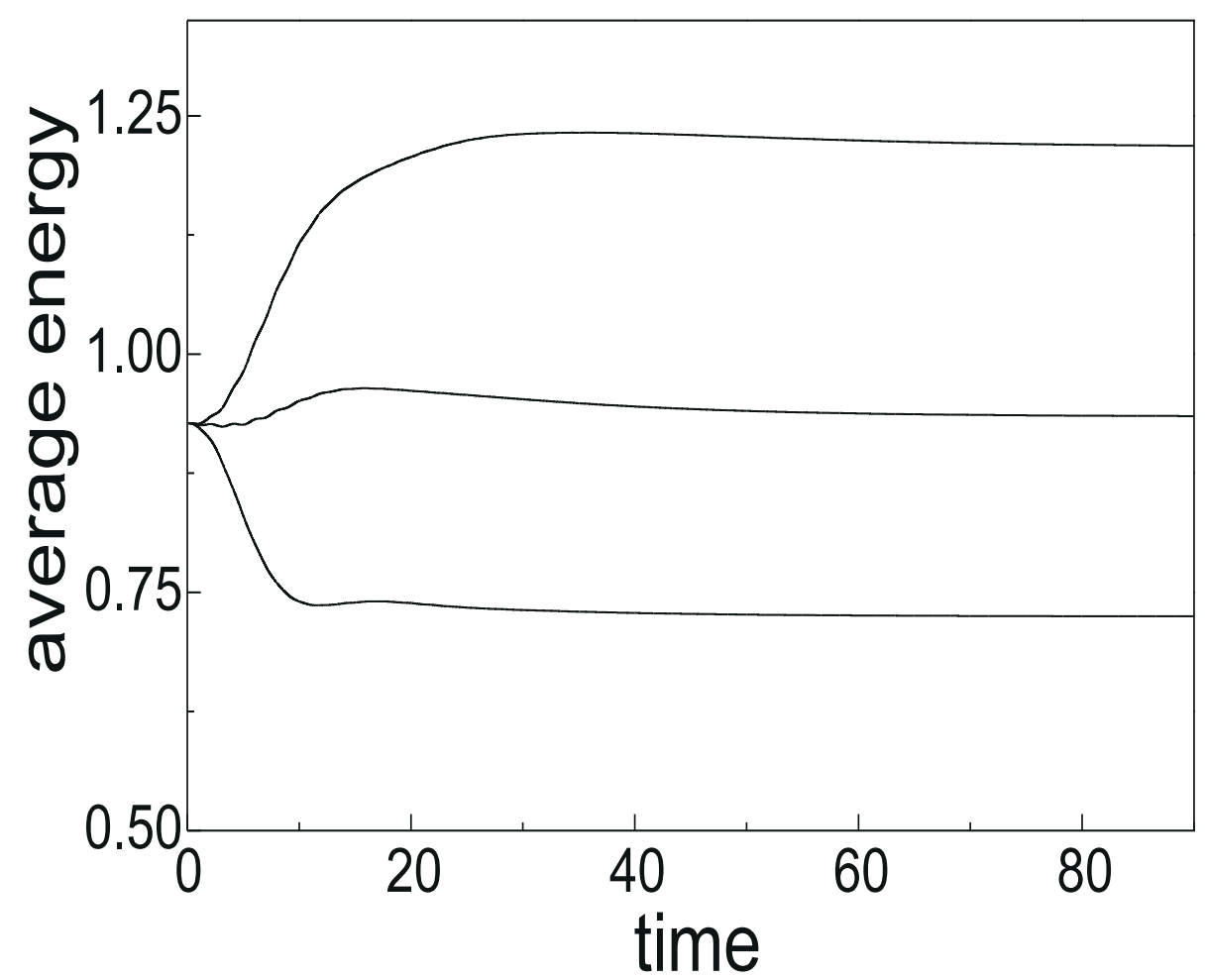

FIG. 4: Evolution of the energy expectation value of the shuttle over time (in units of the oscillator period) as a function of the damping, $\gamma$. From top to bottom, the curves correspond to $\gamma=0.015,0.025$ and 0.05 , with $\varepsilon_{b}=0.86, V=0.5, \alpha=0.2$, $x_{0}=5$ and $\Gamma=0.05$. 


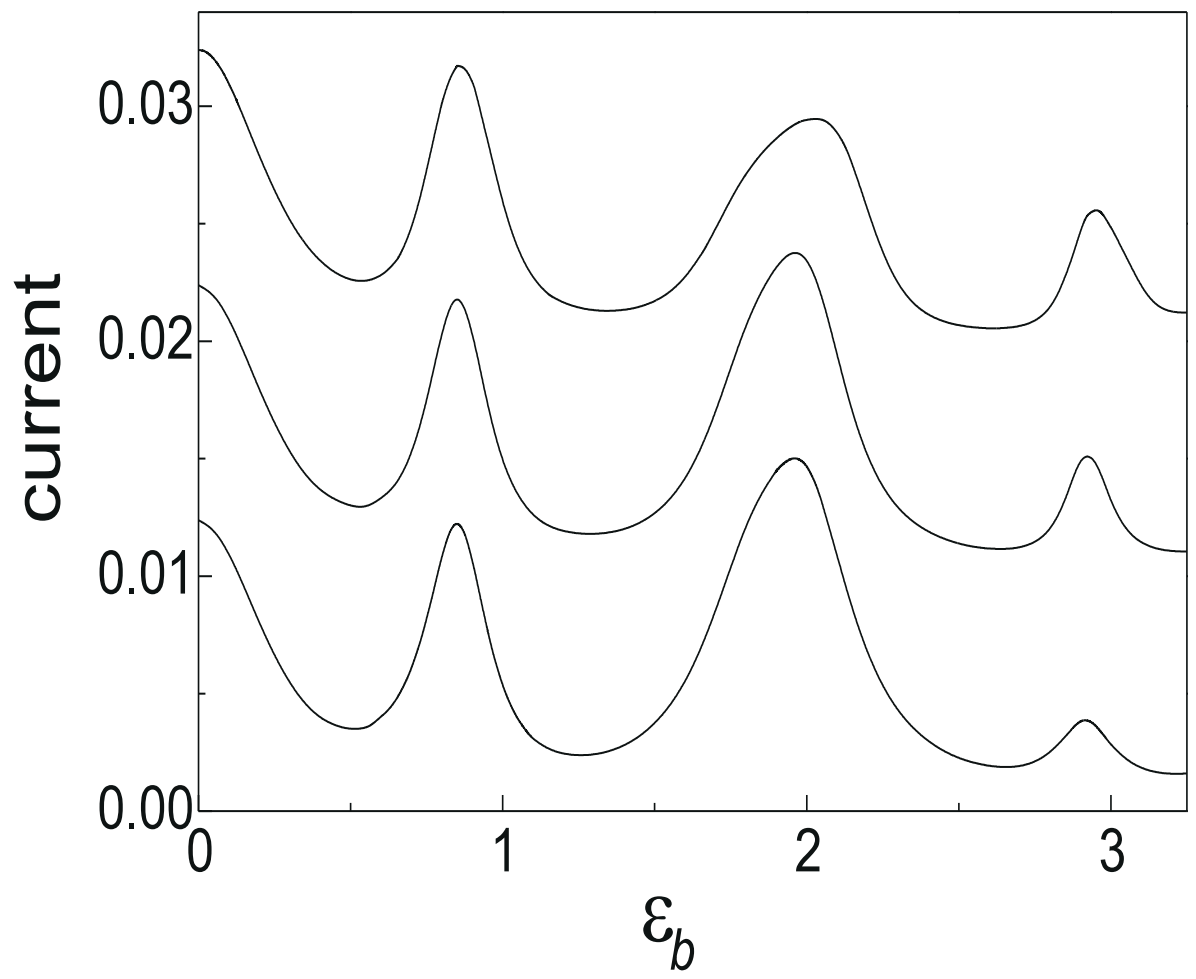

FIG. 5: Effect of the oscillator damping on the current. The curves have been displaced for clarity, from top to bottom they correspond to $\gamma=0.005,0.025$ and 0.05 , with $V=0.5, \alpha=0.2, x_{0}=5$ and $\Gamma=0.05$.

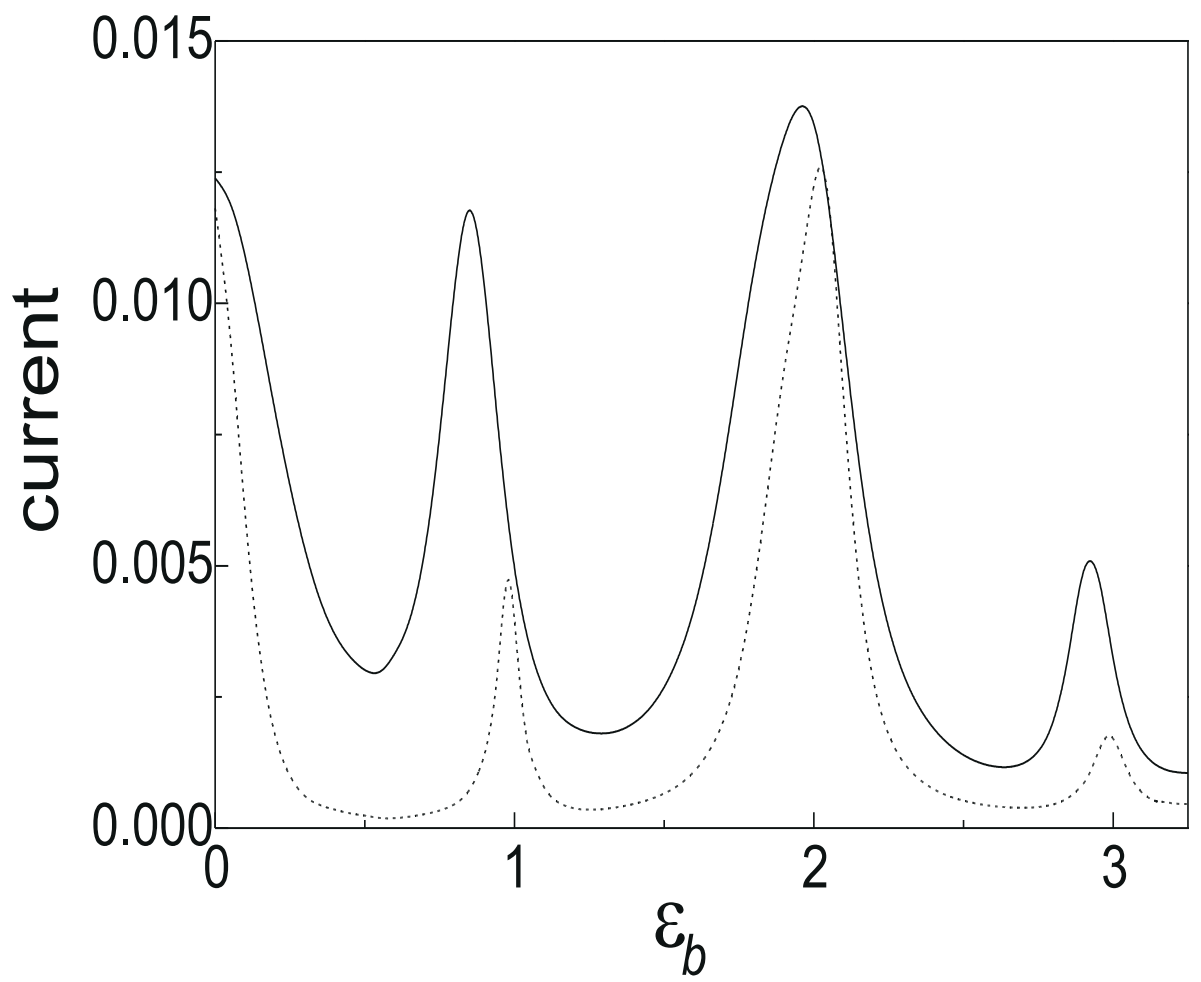

FIG. 6: Steady state current through the shuttle where $V=0.5, \Gamma=0.05, \gamma=0.025, x_{0}=5$ and $\alpha=0.2$ (continuous line) 0.4 (dashed line). 


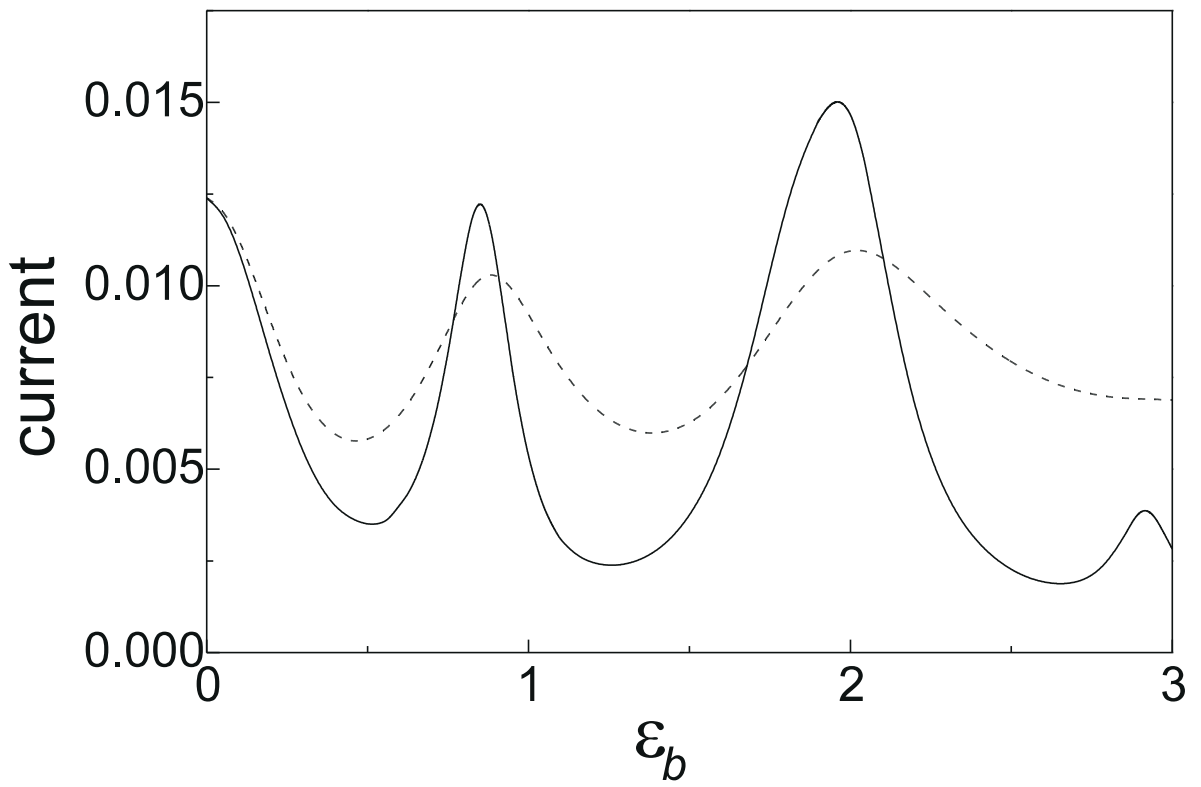

FIG. 7: Steady state current through the shuttle at $k_{\mathrm{B}} T=3$ (dashed line), compared with that at $k_{\mathrm{B}} T=0$ (full line). The parameters are as follows: $V=0.5, \alpha=0.2, \gamma=0.05, x_{0}=5$ and $\Gamma=0.05$. 